\title{
Stability Properties of Neighbourly Random Polytopes
}

\author{
Piotr Mankiewicz • Nicole Tomczak-Jaegermann
}

Received: 22 August 2007 / Revised: 17 April 2008 / Accepted: 17 May 2008 /

Published online: 20 June 2008

(C) Springer Science+Business Media, LLC 2008

\begin{abstract}
We introduce a quantitative parameter measuring $m$-neighbourliness of symmetric convex polytopes in $\mathbb{R}^{k}$. We discuss this parameter for random polytopes generated by subgaussian vectors and show its stability properties.
\end{abstract}

Keywords Symmetric random polytopes $\cdot$ Subgaussian vectors $\cdot m$-Neighbourly polytopes

\section{Introduction}

In this paper we study some geometric properties of convex symmetric polytopes related to the structure of their faces. Neighbourly polytopes, although known to geometers for a long time, recently gained renewed attention, due to their connections to combinatorial problems (see $[5,13]$ and references therein). The classical notion of $m$-neighbourly polytopes, defined in terms of disjointness of convex hulls of appropriate subsets of vertices (see Sect. 2 below), does not provide an information on extent of $m$-neighbourliness of the polytope. We introduce a quantitative property measuring how neighbourly an $m$-neighbourly polytope is. We will study this property for different perturbations of random symmetric polytopes generated

Communicated by Imre Bárány.

Research of P. Mankiewicz was partially supported by KBN Grant no. 1 P03A 01527. N. Tomczak-Jaegermann holds the Canada Research Chair in Geometric Analysis.

P. Mankiewicz (凶)

Institute of Mathematics, PAN, Śniadeckich 8, P.O. Box 21, 00-956 Warsaw 10, Poland e-mail: piotr@impan.gov.pl

N. Tomczak-Jaegermann

Department of Mathematical and Statistical Sciences, University of Alberta, Edmonton, T6G 2G1 Alberta, Canada

e-mail: nicole.tomczak@ualberta.ca 
by subgaussian matrices. This makes a connection to a rich theory of random finitedimensional Banach spaces. Such spaces, in particular random quotients of $\ell_{1}^{n}$, s, have been extensively studied over last quarter of a century and led to solutions of several classical problems (cf. [4, 8, 14], see also the survey [9]). Random polytopes (including $+1 /-1$ polytopes) also play a very important role in combinatorics; we mention only a few recent results [1-3].

We shall now briefly describe the results and organization of this paper. In Sect. 2, apart of standard preliminaries, we introduce the quantitative notion of $(m, \alpha)$ neighbourly polytopes. In particular, a symmetric polytope is $m$-neighbourly if and only if it is $(m, \alpha)$-neighbourly for some $\alpha>1$. Subsequently we define related isometric invariants nei $i_{m}(K)$. The main results are presented in Sect. 3. We begin by providing upper estimates for $\alpha$, equivalent to (the same) upper bounds for nei $i_{m}$. It turns out that, with probability close to 1 , symmetric polytopes determined by subgaussian vectors have invariants nei $m(K)$ of maximal order (up to a logarithmic factor) for every $m$ in the admissible range. It is surprising that this property is pretty stable for a random polytope $K$. In particular, for any not-too-large family of sufficiently nontrivial operators $T_{\mu}$, this property holds simultaneously for all $T_{\mu}(K)$ (Theorem 3.2). The same holds when the fixed family $T_{\mu}$ is replaced by a family of projections annihilating some subsets of vertices (Theorem 3.3), or when the polytope $K$ is replaced by the convex hull of itself and its random rotation (Theorem 3.4). In Sect. 4 we develop, in language of convex analysis, criteria for the $(m, \alpha)$-neighbourliness. This approach follows the method presented in [10] (Chap. 4). In the next Sect. 5 we prove Proposition 3.1 on upper bounds for $\operatorname{nei}_{m}(K)$. In Sect. 6 we state and prove the technical probabilistic fact (Proposition 6.1) that underlies all main results of our paper. In the last Sect. 7 we deduce Theorems 3.2, 3.3, and 3.4 from Proposition 6.1.

\section{Preliminaries}

We use the standard notation. In particular, $\langle\cdot, \cdot\rangle$ and $|\cdot|$ stand for the standard scalar product and the Euclidean norm on $\mathbb{R}^{k}$, while $\left\{e_{1}, e_{2}, \ldots, e_{k}\right\}$ denotes the standard unit vector basis in $\mathbb{R}^{k}$. By $B_{2}^{k}$ and $S^{k-1}$ we denote the Euclidean unit ball and unit sphere, respectively. $B_{1}^{k}$ stands for the $k$-dimensional cross-polytope, that is, the unit ball in the $\ell_{1}$ norm on $\mathbb{R}^{k}$. The space of linear operators acting from $\mathbb{R}^{n}$ into $\mathbb{R}^{k}$ is denoted by $L\left(\mathbb{R}^{n}, \mathbb{R}^{k}\right)$.

We study some geometric properties of convex symmetric polytopes in $\mathbb{R}^{k}$, that is, sets of the form

$$
K=\operatorname{conv}\left\{ \pm x_{1}, \pm x_{2}, \ldots, \pm x_{n}\right\}
$$

where $n>k>2$ and $x_{i} \in \mathbb{R}^{k}$ for $i=1,2, \ldots, n$. By $\mathcal{V}(K)$ we denote the set of vertices of $K$. By $\mathcal{V}_{m}(K)$ for $1 \leq m<k$ we denote the family of all nonempty subsets of $\mathcal{V}(K)$ with cardinality less than or equal to $m$, not containing a pair of antipodal vertices.

Recall that a convex symmetric polytope $K$ in $\mathbb{R}^{k}$ is said to be $m$-neighbourly, where $2 \leq m<k$, if and only if every subset $V \in \mathcal{V}_{m}(K)$ is a vertex set for a face 
of $K$. In particular this means that

$$
\operatorname{conv} V \cap \operatorname{conv}\{\mathcal{V}(K) \backslash V\}=\varnothing
$$

for every $V \in \mathcal{V}_{m}(K)$. By a standard compactness argument this implies that there exists $\alpha>1$ such that

$$
\operatorname{conv} V \cap \alpha \operatorname{conv}\{\mathcal{V}(K) \backslash V\}=\emptyset
$$

for every $V \in \mathcal{V}_{m}(K)$. Let us observe that for an arbitrary convex symmetric polytope $K$ in $\mathbb{R}^{k}$ (not necessary $m$-neighbourly for any $m \geq 2$ ), a similar argument shows that there exists $\alpha>1$ such that (2.2) holds for every singleton $\{x\} \in \mathcal{V}(K)$.

Motivated by the above, we shall say that a convex symmetric polytope is $m$-neighbourly with parameter $\alpha$ for $1 \leq m<k$ and $\alpha>1$, or shortly $(m, \alpha)$ neighbourly if (2.2) holds for every $V \in \mathcal{V}_{m}(K)$. Finally we define the $m$-neighbourliness constant $\operatorname{nei}_{m}(K)$ of an $m$-neighbourly polytope $K$ by

$$
\operatorname{nei}_{m}(K)=\sup \{\alpha \mid K \text { is }(m, \alpha) \text {-neighbourly }\} \text {. }
$$

Additionally we put nei ${ }_{m}(K)=1$ if $K$ is not $m$-neighbourly.

Note that in the definition above, we allow the case $m=1$ as well. In what follows, for technical reasons, we will work with the parameters $\alpha$ rather then with the invariants nei $_{m}$ themselves.

It is known that a convex symmetric polytope $K \subset \mathbb{R}^{k}$ with $|\mathcal{V}(K)| \geq 2(k+2)$ may be at most $\lfloor(k+1) / 3\rfloor$-neighbourly [12]. Note that nei $i_{m}(K)$ is a non-increasing function of $m$. Moreover if $2 n=|\mathcal{V}(K)|>2 k$, then $\operatorname{nei}_{m}(K)$ is finite for every $m$. Indeed, note that $\operatorname{nei}_{1}(K) \geq \operatorname{nei}_{m}(K)$ and let $\mathcal{V}(K)=\left\{ \pm x_{1}, \pm x_{2}, \ldots, \pm x_{n}\right\}$. After possible reordering, we may assume that $x_{1}$ can be written as linear combination of $\left\{x_{2}, \ldots, x_{n}\right\}$, which yields

$$
x_{1} \in \alpha \operatorname{conv}\left\{ \pm x_{2}, \pm x_{3}, \ldots, \pm x_{n}\right\}
$$

for a suitable large $\alpha>1$. Thus nei ${ }_{1}(K) \leq \alpha$. Clearly nei ${ }_{m}(K)=$ nei $_{m}(S K)$ for every linear isomorphism $S \in L\left(\mathbb{R}^{k}, \mathbb{R}^{k}\right)$. In general, if for some $T \in L\left(\mathbb{R}^{k}, \mathbb{R}^{k}\right)$, one has $|\mathcal{V}(K)|=|\mathcal{V}(T K)|$, then

$$
\operatorname{nei}_{m}(K) \geq \operatorname{nei}_{m}(T K) .
$$

With a polytope $K=\operatorname{conv}\left\{ \pm x_{1}, \pm x_{2}, \ldots, \pm x_{n}\right\} \subset \mathbb{R}^{k}$, one can associate a $k \times n$ matrix $T$ whose columns are the vectors $x_{1}, x_{2}, \ldots, x_{n}$. We shall often identify this matrix with the linear operator from $\mathbb{R}^{n}$ to $\mathbb{R}^{k}$ defined by

$$
T\left(e_{i}\right)=x_{i} \quad \text { for } i=1,2, \ldots, n \text {. }
$$

Conversely, to every operator $T: \mathbb{R}^{n} \rightarrow \mathbb{R}^{k}$, in a natural way there corresponds the polytope

$$
K=\operatorname{conv}\left\{ \pm T\left(e_{1}\right), \pm T\left(e_{2}\right), \ldots, \pm T\left(e_{n}\right)\right\}
$$


We now recall a few definitions related to subgaussian vectors and matrices. For a random variable $h$ defined on a probability space $(\Omega, \mathbb{P})$ and $0<p<\infty$, let

$$
\|h\|_{p}=\left(\int_{\Omega}|h|^{p} d \mathbb{P}\right)^{1 / p} .
$$

A random variable $\gamma$ is called subgaussian if there exists $\beta<\infty$ such that

$$
\|\gamma\|_{2 k} \leq \beta\|g\|_{2 k} \quad \text { for } k=1,2, \ldots,
$$

where $g$ denotes the $\mathcal{N}(0,1)$ Gaussian variable. We shall refer to the infimum over all $\beta$ satisfying (2.3) as the subgaussian constant of $\gamma$. In this paper we consider only symmetric subgaussian random variables, but the results are valid in a more general case of subgaussian random variables of mean zero. In this case appropriate probabilistic estimates (with slightly worse constants) are obtained from the corresponding case of symmetric random variables by a symmetrization procedure standard in probability.

Let us note that there are other equivalent frequently used definitions of subgaussian variables, for example, in terms of the Orlicz function $\psi_{2}$ or by tail distribution. We refer the reader to, e.g., [6] for some further comments.

A random vector $x \in \mathbb{R}^{k}$ with independent subgaussian entries will be called a subgaussian vector.

The main examples of subgaussian random variables are Gaussian random variables, Bernoulli random variables, or any bounded random variables.

We shall work with random polytopes generated by matrices $\Gamma$ with i.i.d. symmetric subgaussian entries. The most important examples of such polytopes, apart from the Gaussian ones, are the so called $+1 /-1$ polytopes given by matrices with independent Bernoulli entries. Equivalently, these are symmetric random polytopes of the form conv $\left\{ \pm x_{1}, \ldots, \pm x_{n}\right\}$ in $\mathbb{R}^{k}$, where the $x_{i}$ 's are independent uniformly distributed vertices of the combinatorial cube $\{-1,1\}^{k}$.

In what follows the letters $C, c, c^{\prime}, \ldots$ denote numerical constants, whose values may be different in different paragraphs. By $\log (\cdot)$ we denote the logarithm to the base $e$.

\section{Results}

The following proposition provides the upper estimates for $\alpha$ for $(m, \alpha)$-symmetric neighbourly polytopes. Namely we have the following:

Proposition 3.1 Let $1<k<n$, and let $K \subset \mathbb{R}^{k}$ be an $(m, \alpha)$-neighbourly convex symmetric polytope with $|\mathcal{V}(K)|=2 n$. Then

(i) $\alpha \leq(k+1-m) / m$.

(ii) If $n \geq k(k+1) / 2+m$, then $\alpha \leq \sqrt{k / m}$. 
For a "typical" random polytope $K$ generated by independent subgaussian vectors, one can prove, by suitably adopting the argument presented in [10], that it is $(m, \alpha)$ symmetric neighbourly for relatively large $m$ and $\alpha$. The theorem below states that a similar fact holds "typically" for all polytopes of the form $T_{\mu}(K)$, where $K$ is as above, and $\left\{T_{\mu}\right\}$ is a family of sufficiently nontrivial operators in $L\left(\mathbb{R}^{k}, \mathbb{R}^{k}\right)$ with controlled cardinality. More precisely:

Theorem 3.2 There exist numerical constants $c_{1}, c_{2}>0$ such that the following holds. Let $\gamma$ be a symmetric subgaussian random variable with $\operatorname{Var} \gamma \geq 1$ and the subgaussian constant $\beta$. Let $k<n$ be positive integers, and let $\Gamma$ be a $k \times n m a$ trix with independent entries distributed according to $\gamma$. Set $K=\operatorname{conv}\left\{ \pm \Gamma\left(e_{i}\right) \mid i=\right.$ $1,2, \ldots, n\}$ and let $\left\{T_{\mu}\right\}_{\mu \leq N}$ be a family of linear operators in $\mathbb{R}^{k}$ with $\operatorname{rank} T_{\mu} \geq k / 4$ for $\mu=1,2, \ldots, N$. With probability greater than

$$
1-N \exp \left(-c_{1} k / \beta^{4}\right)
$$

the polytopes $T_{\mu} K$ for $\mu=1,2, \ldots, N$ are $(m, \alpha)$-neighbourly for every pair $(m, \alpha)$, where $\alpha>1$, and $m \geq 1$ is an integer satisfying

$$
\alpha^{2} m \leq \frac{c_{2} k}{\beta^{6} \log \left(n \beta^{4} / c_{2} k\right)},
$$

and $\left|\mathcal{V}\left(T_{\mu} K\right)\right|=2 n$. In particular,

$$
\sqrt{m} \operatorname{nei}_{m}\left(T_{\mu} K\right) \geq \frac{1}{\beta^{3}}\left(\frac{c_{2} k}{\log \left(n \beta^{4} / c_{2} k\right)}\right)^{1 / 2}
$$

for every $m$ satisfying

$$
m<\frac{c_{2} k}{\beta^{6} \log \left(n \beta^{4} / c_{2} k\right)} .
$$

Remark It follows from the proof of Theorem 3.2 that the constant $c_{2}$ is small. Note that the condition in the theorem on the $(m, \alpha)$-neighbourliness of polytopes in question requires that the expression on the right-hand side of (3.1) is greater than 1 , which in turn yields that $k>\beta^{6} / c_{2}$.

Applying Theorem 3.2 to the Bernoulli random variables leads to so-called random $+1 /-1$ polytopes. Then considering just one operator equal to the identity, we conclude that for any pair $(m, \alpha)$ satisfying (3.1), with probability close to 1 , a random $+1 /-1$ polytope $K$ is $(m, \alpha)$-neighbourly. In particular, setting $\mathcal{V}(K)=$ $\left\{u_{1}, \ldots, u_{2 n}\right\}$, we get

$$
u_{i} \notin\left(\frac{c k}{\log (C n / k)}\right)^{1 / 2} \operatorname{conv}\left\{u_{j} \mid j \neq i\right\} \quad \text { for } i=1, \ldots, 2 n,
$$

and, more generally,

$$
\operatorname{conv}\left\{u_{i} \mid u_{i} \in V\right\} \cap \frac{1}{\sqrt{m}}\left(\frac{c k}{\log (C n / k)}\right)^{1 / 2} \operatorname{conv}\left\{u_{j} \mid u_{j} \notin V\right\}=\emptyset
$$


for all $V \in \mathcal{V}_{m}(K)$ and all $1 \leq m<c k / \log (C n / k)$. (Here $C>1$ and $c>0$ are numerical constants.) Note that the case of $m=1$ is a simple consequence of a result from [6] (Corollary 4.3), while the case of $m=m_{0}=[\mathrm{ck} / \log (\mathrm{Cn} / \mathrm{k})]$ was proved in [10] (for a more general case of polytopes generated by subgaussian vectors). Therefore, formula (3.2) provides, in a sense, the whole "scale" of results for arbitrary $1 \leq m \leq m_{0}$.

In Theorem 3.2 the operators $T_{\mu}$ remained fixed for all random polytopes of the form $K=\Gamma\left(B_{1}^{n}\right)$. In the theorem below the family of considered projections depends on the random polytope in question. Namely, let $K=\operatorname{conv}\left\{ \pm x_{i} \mid i=1,2, \ldots, n\right\}$. For each subset $\sigma \subset\{1,2, \ldots, n\}$, we let $P_{\sigma}=P_{\sigma, K}$ be the orthogonal projection with $\operatorname{ker} P_{\sigma}=\operatorname{lin}\left\{x_{i} \mid i \in \sigma\right\}$.

Theorem 3.3 There exist numerical constants $c_{1}, c_{2}>0$ such that the following holds. Let $\gamma$ be a symmetric subgaussian random variable with $\operatorname{Var} \gamma \geq 1$ and the subgaussian constant $\beta$. Let $k<n$ be positive integers. Let $\Gamma$ and $K$ be as in Theorem 3.2 , and let $1 \leq \ell \leq 3 k / 4$. Assume that $k<n-\ell$. With probability greater than

$$
1-\left(\begin{array}{l}
n \\
\ell
\end{array}\right) \exp \left(-c_{1} k / \beta^{4}\right)
$$

polytopes $P_{\sigma} K$, for all $\sigma$ with $|\sigma|=\ell$ are $(m, \alpha)$-neighbourly for every pair $(m, \alpha)$, where $\alpha>1$, and $m \geq 1$ is an integer satisfying

$$
\alpha^{2} m \leq \frac{c_{2} k}{\beta^{6} \log \left(n \beta^{4} / c_{2} k\right)},
$$

and $\left|\mathcal{V}\left(P_{\sigma} K\right)\right|=2(n-\ell)$. In particular,

$$
\sqrt{m} \operatorname{nei}_{m}\left(P_{\sigma} K\right) \geq \frac{1}{\beta^{3}}\left(\frac{c_{2} k}{\log \left(n \beta^{4} / c_{2} k\right)}\right)^{1 / 2}
$$

for every $m$ satisfying

$$
m<\frac{c_{2} k}{\beta^{6} \log \left(n \beta^{4} / c_{2} k\right)} .
$$

Remark The requirement that the probability in theorems above is positive imposes restrictions on how large $N$ and $\ell$ may be. In particular, if we choose $\ell<$ $c k / \log (c n / k)$ for suitable $c>0$, then the probability in Theorem 3.3 is exponentially close to 1 .

Let $v_{k}$ denote the normalized Haar measure on the orthogonal group $O(k)$, and let $\tilde{\mathbb{P}}=\mathbb{P} \times v_{k}$ be the product probability on $\Omega \times O(k)$. Then we have:

Theorem 3.4 There exist numerical constants $c_{1}, c_{2}>0$ such that the following holds. Let $\gamma$ be a symmetric subgaussian random variable with $\operatorname{Var} \gamma \geq 1$ and the subgaussian constant $\beta$. Let $k<n$ be positive integers. Let $\Gamma$ and $K$ be as in Theorem 3.2, and let $K_{U}=\operatorname{conv}\{K \cup U(K)\}$ for $U \in O(k)$. With probability $\tilde{\mathbb{P}}$ greater 
than

$$
1-\exp \left(-c_{1} k / \beta^{4}\right)
$$

the polytope $K_{U}$ is $(m, \alpha)$-neighbourly for every integer $m \geq 1$ and $\alpha>1$ satisfying

$$
\alpha^{2} m \leq \frac{c_{2} k}{\beta^{6} \log \left(n \beta^{4} / c_{2} k\right)},
$$

and $\left|\mathcal{V}\left(K_{U}\right)\right|=4 n$. In particular,

$$
\sqrt{m} \operatorname{nei}_{m}\left(K_{U}\right) \geq \frac{1}{\beta^{3}}\left(\frac{c_{2} k}{\log \left(n \beta^{4} / c_{2} k\right)}\right)^{1 / 2}
$$

for every $m$ satisfying

$$
m<\frac{c_{2} k}{\beta^{6} \log \left(n \beta^{4} / c_{2} k\right)} .
$$

Remark Recall that by the main result in [7] on singular values of rectangular matrices (Theorem 3.1 and Remark 4 following it) one has: if $n \geq(1+\delta) k$ for some $\delta>0$, then, with probability exponentially (in $n$ ) close to $1, K$ contains $\rho B_{2}^{k}$ for some $\rho>0$ depending on $\delta$ only. Using (6.2) with, e.g., $u=3 \beta$, and applying Lemma 5.1 below, we conclude that with probability exponentially (in $k$ ) close to 1 , the polytopes $P K$ for every rank $P \geq k / 4$ are not $(m, \alpha)$-neighbourly for $\alpha \geq \bar{c} \sqrt{k / m}$. Thus, in the theorems above, for $m$ in the admissible range and $n \geq k(k+1) / 2+m$, the values of $\alpha$ are optimal up to a logarithmic factor.

\section{Criteria for Neighbourliness}

This section discusses simple analytic conditions sufficient for the $(m, \alpha)$-neighbourliness of polytopes. This is a slight modification of arguments from [10].

For a fixed $\alpha>1$ and an arbitrary pair of disjoint subsets $I, J \subset\{1,2, \ldots, n\}$, we shall consider the cone

$$
C_{I, J, \alpha}=\left\{\left(t_{i}\right) \in \mathbb{R}^{n}\left|-\sum_{i \in I} t_{i}+\sum_{j \in J} t_{j}+\alpha^{-1} \sum_{i \notin I \cup J}\right| t_{i} \mid \leq 0\right\} .
$$

By $C_{I, J, \alpha}^{0}$ we denote the interior of $C_{I, J, \alpha}$.

Lemma 4.1 Let $\Gamma$ be a $k \times n$ matrix with $1<k<n$. For any $\alpha>1$ and disjoint subsets $I, J$ of $\{1,2, \ldots, n\}$, the following conditions are equivalent:

(i) $\operatorname{ker} \Gamma \cap C_{I, J, \alpha}^{0}=\emptyset$;

(ii) there exists $z_{0} \in \mathbb{R}^{k}$ such that

$$
\begin{aligned}
&\left\langle z_{0}, \Gamma e_{i}\right\rangle=\left\langle z_{0},-\Gamma e_{j}\right\rangle=1 \quad \text { for } i \in I, j \in J, \quad \text { while } \\
&\left|\left\langle z_{0}, \Gamma e_{i}\right\rangle\right| \leq \alpha^{-1} \quad \text { for } i \notin I \cup J .
\end{aligned}
$$


Proof Indeed, assuming (i), by a separation argument there is $y \in \mathbb{R}^{n}$ with $y \perp \operatorname{ker} \Gamma$ and $\langle y, x\rangle>0$ for every $x \in C_{I, j, \alpha}^{0}$ (hence also $\langle y, x\rangle \geq 0$ for every $x \in C_{I, j, \alpha}$ ). Note that $e_{i} \in C_{I, J, \alpha}^{0}$ and $\pm\left(e_{i}-e_{i^{\prime}}\right) \in C_{I, J, \alpha}$ for every $i, i^{\prime} \in I$. Thus

$$
\left\langle y, e_{i}\right\rangle=\left\langle y, e_{i^{\prime}}\right\rangle>0
$$

for every $i, i^{\prime} \in I$. Similarly we get $\left\langle y, e_{j}\right\rangle=\left\langle y, e_{j^{\prime}}\right\rangle<0$ for every $j, j^{\prime} \in J$. Finally, since $\pm\left(e_{i}+e_{j}\right) \in C_{I, J, \alpha}$, we have

$$
\left\langle y, e_{i}\right\rangle=\left\langle y,-e_{j}\right\rangle>0
$$

for every $i \in I$ and $j \in J$. Multiplying $y$ by a suitable constant $c>0$, we get

$$
\left\langle c y, e_{i}\right\rangle=\left\langle c y,-e_{j}\right\rangle=1 \quad \text { for every } i \in I \text { and } j \in J \text {. }
$$

On the other hand, for every $i \in I$ and every $j \notin I \cup J$, the vectors $e_{i} \pm \alpha e_{j}$ belong to $C_{I, J, \alpha}$, which yields

$$
\left|\left\langle c y, e_{j}\right\rangle\right| \leq 1 / \alpha .
$$

Finally, note that since $y \perp \operatorname{ker} \Gamma$, there exists $z_{0} \in \mathbb{R}^{k}$ such that $\Gamma^{*} z_{0}=c y$, which means that $\left\langle z_{0}, \Gamma e_{i}\right\rangle=\left\langle c y, e_{i}\right\rangle$ for every $i=1,2, \ldots, n$.

Conversely, if there exists $z_{0}$ satisfying (ii), then $y=\Gamma^{*} z_{0}$ satisfies $y \perp \operatorname{ker} \Gamma$ and $\langle y, x\rangle>0$ for every $x \in C_{I, j, \alpha}^{0}$. Hence $\operatorname{ker} \Gamma \cap C_{I, J, \alpha}^{0}=\emptyset$.

Remark Note that Lemma 4.1 in particular yields that if $\operatorname{ker} \Gamma$ is disjoint with $C_{I, J, \alpha}^{0}$, then $\operatorname{conv}\left\{\Gamma e_{1},-\Gamma e_{j} \mid i \in I, j \in J\right\}$ is a face of the polytope $K=\operatorname{conv}\left\{ \pm \Gamma e_{i} \mid i=\right.$ $1,2, \ldots, n\}$.

Let $K=\operatorname{conv}\left\{ \pm \Gamma e_{i} \mid i=1,2, \ldots, n\right\}$, and let $V=\left\{\Gamma e_{i},-\Gamma e_{j} \mid i \in I\right.$ and $j \in$ $J\} \in \mathcal{V}_{m}(K)$. The following corollary is a direct consequence of Lemma 4.1.

Corollary 4.2 In the above notation, conv $V$ is a face that, for every $1<\alpha^{\prime}<\alpha$, is disjoint from the $\alpha^{\prime}$ multiple of the convex hull of the remaining vertices

$$
\alpha^{\prime} \operatorname{conv}\{\mathcal{V}(K) \backslash V\}
$$

if and only if $\operatorname{ker} \Gamma \cap C_{I, J, \alpha}^{0}=\emptyset$. In particular, for every $i=1,2, \ldots, n$, the condition $\operatorname{ker} \Gamma \cap C_{\{i\}, \emptyset, \alpha}^{0}=\emptyset$ implies that $\Gamma e_{i}$ is a vertex for the polytope $K$ (hence $-\Gamma e_{i}$ is a vertex as well).

This means that to prove that $K=\operatorname{conv}\left\{ \pm \Gamma e_{i}\right\}$ is $(m, \alpha)$-neighbourly polytope, it is sufficient to show that all cones $C_{I, J, \alpha}^{0}$ with $1 \leq|I|+|J| \leq m$ are disjoint from $\operatorname{ker} \Gamma$. That is, we are going to prove that

$$
\bigcup_{1 \leq|I|+|J| \leq m} C_{I, J, \alpha}^{0} \cap \operatorname{ker} \Gamma=\emptyset .
$$


In order to simplify control of the union of the cones, one more idea from [10] is useful. For every $1 \leq l \leq n$, we set

$$
U_{l}=\left\{x \in S^{n-1}|| \operatorname{support} x \mid \leq l\right\} .
$$

(For $x=\left(a_{1}, \ldots, a_{n}\right) \in \mathbb{R}^{n}$, we let support $x=\left\{i \mid a_{i} \neq 0\right\}$.)

Lemma 4.3 Let $1 \leq m \leq n$ and $\alpha>1$. Then

$$
\begin{aligned}
& S^{n-1} \cap \bigcup_{1 \leq|I|+|J| \leq m} C_{I, J, \alpha} \subset 2 \alpha \sqrt{m} B_{1}^{n} \cap S^{n-1} \\
& \subset 2 \operatorname{conv} U_{4 \alpha^{2} m} \cap S^{n-1} .
\end{aligned}
$$

Proof The first inclusion follows from the fact that for all $I$ and $J$, by the definition of $C_{I, J, \alpha}$, one has $\|x\|_{1} \leq 2 \alpha \sqrt{|I|+|J|}$ for every $x \in C_{I, J, \alpha} \cap S^{n-1}$.

To prove the second inclusion, note that

$$
2 \alpha \sqrt{|I|+|J|} B_{1} \cap S^{n-1} \subset 2 \alpha \sqrt{m}\left(B_{1} \cap \frac{1}{2 \alpha \sqrt{m}} S^{n-1}\right) .
$$

By Lemma 3.2 in [10] (or Lemma 3.7 in [11]), for every $x \in \mathbb{R}^{n}$, we have for $l=4 \alpha^{2} m$,

$$
\sup \langle x, z\rangle \leq 2 \sup _{w \in U_{l}}\langle x, w\rangle
$$

where the first supremum is taken over $z \in 2 \alpha \sqrt{m} B_{1}^{n} \cap S^{n-1}$. By the separation argument, it follows that

$$
2 \alpha \sqrt{m} B_{1} \cap S^{n-1} \subset\left(2 \operatorname{conv} U_{4 \alpha^{2} m}\right) \cap S^{n-1} .
$$

In view of these results, we have the following obvious corollary, which is stated in terms of $m, n, \alpha$, and $\operatorname{ker} \Gamma$ only. Recall that $\Gamma$ is a $k \times n$ matrix with $1<k<n$ and note that $k$ is related to $n$ by the condition

$$
k+\operatorname{dim} \operatorname{ker} \Gamma \geq n
$$

Corollary 4.4 In the notation above, let $m<n$ be positive integers, $\alpha>1$ and the matrix $\Gamma$ be such that

$$
\operatorname{ker} \Gamma \cap\left(2 \alpha \sqrt{m} B_{1}^{n} \cap S^{n-1}\right)=\emptyset .
$$

Then the polytope $K=\operatorname{conv}\left\{ \pm \Gamma e_{i}\right\}$ is $(m, \alpha)$-neighbourly. Moreover $\mathcal{V}(K)=$ $\left\{ \pm \Gamma e_{i}\right\}$. 


\section{Proof of Proposition 3.1}

Proof (i) Let $\mathcal{V}(K)=\left\{ \pm x_{1}, \pm x_{2}, \ldots, \pm x_{n}\right\}$. Consider a $k \times n$ matrix $A$ whose columns are vectors $x_{i}(i=1, \ldots, n)$ and view $A$ as a linear operator in $L\left(\mathbb{R}^{n}, \mathbb{R}^{k}\right)$. Since $\operatorname{dim} \operatorname{ker} A \geq n-k$, there exists $0 \neq x_{0} \in \operatorname{ker} A$ of the form

$$
x_{0}=\left(s_{1}, s_{2}, \ldots, s_{k+1}, 0,0, \ldots, 0\right) .
$$

By relabeling the coordinates, we may assume without lost of generality that $\left|s_{1}\right| \geq$ $\left|s_{2}\right| \geq \cdots \geq\left|s_{k+1}\right|$. Now we consider the first $m$ coordinates and divide them into the nonnegative and the negative ones. Namely, set $I=\left\{i \leq m \mid s_{i} \geq 0\right\}$ and $J=$ $\left\{j \leq m \mid s_{j}<0\right\}$. Note that $\sum_{i \in I} s_{i}-\sum_{j \in J} s_{j} \geq m\left|s_{m}\right|$. Pick an arbitrary $\tilde{\alpha}>\alpha_{0}=$ $(k+1-m) / m$. Then

$$
-\sum_{i \in I} s_{i}+\sum_{j \in J} s_{j}+\tilde{\alpha}^{-1} \sum_{\nu>m}\left|s_{\nu}\right|<\frac{k+1-m}{\alpha_{0}}\left|s_{m}\right|-m\left|s_{m}\right|=0 .
$$

This yields that $x_{0} \in \operatorname{ker} A \cap C_{I, J, \tilde{\alpha}}^{0}$. Hence, by Corollary 4.2 there is $1<\alpha^{\prime}<\tilde{\alpha}$ such that the face $\operatorname{conv}\left\{A e_{i}, A\left(-e_{j}\right) \mid i \in I, j \in J\right\}$ has a nonempty intersection with

$$
\alpha^{\prime} \operatorname{conv}\left\{A\left(-e_{i}\right), A e_{j}, A e_{v} \mid i \in I, j \in J, v>m\right\} .
$$

Since this is true for an arbitrary $\tilde{\alpha}>\alpha_{0}$, it follows that $\alpha \leq \alpha_{0}$ which completes the proof of (i), by the definition of $\alpha_{0}$.

For the proof of part (ii) we need the following lemma

Lemma 5.1 Let $K \subset \mathbb{R}^{k}$ be an $(m, \alpha)$-neighbourly polytope with $\mathcal{V}(K)=\left\{ \pm x_{1} \mid i=\right.$ $1,2, \ldots, n\}$. Assume that there exists $I \subset\{1,2, \ldots, n\}$ with $|I|=m<k$ such that

$$
(1 / \sqrt{k}) B_{2}^{k} \subset \operatorname{conv}\left\{ \pm x_{j} \mid j \notin I\right\}:=\tilde{K} .
$$

Then $\alpha \leq a \sqrt{k / m}$, where $a=\max _{i \in I}\left|x_{i}\right|$.

Proof Clearly $K_{0}=\operatorname{conv}\left\{x_{i} \mid i=1,2, \ldots, n\right\}$ is a (nonsymmetric) $m$-neighbourly polytope. Hence the set of vertices $\left\{x_{i}\right\}_{i \in I}$ is affine independent (see, e.g., [5, p. 122]). Let $E$ be the linear subspace spanned by $\left\{x_{i}\right\}_{i \in I}$. Thus $\operatorname{dim} E=m$. Consider $K_{I}=$ $\operatorname{conv}\left\{ \pm x_{i} \mid i \in I\right\}$. Let $\mathcal{E} \subset E$ be the ( $m$-dimensional) ellipsoid of maximal volume contained in $K_{I}$. Note that $K_{I} \subset E$ is linearly isomorphic to $B_{1}^{m}$ and recall that $(1 / \sqrt{m}) B_{2}^{m}$ is the ellipsoid of maximal volume contained in $B_{1}^{m}$. Since the ratio of volumes is invariant under linear isomorphisms, we infer that

$$
\frac{\operatorname{vol}_{m} \mathcal{E}}{\operatorname{vol}_{m} K_{I}}=\frac{\operatorname{vol}_{m}(1 / \sqrt{m}) B_{2}^{m}}{\operatorname{vol}_{m} B_{1}^{m}} .
$$

On the other hand, by Hadamard's inequality $\operatorname{vol}_{m} K_{I} \leq \operatorname{vol}_{m} a B_{1}^{m}$, hence $\operatorname{vol}_{m} \mathcal{E} \leq$ $\operatorname{vol}_{m}(a / \sqrt{m}) B_{2}^{m}$. Since $\mathcal{E}$ has the maximal volume among all ellipsoids inscribed in $K_{I}$, it follows that $(a / \sqrt{m}) B_{2}^{m}$ is not contained in the relative interior of $K_{I}$. Thus 
there exists a facet $F$ of $K_{I}$ such that $F \cap(a / \sqrt{m}) B_{2}^{k} \neq \emptyset$, and therefore $a \sqrt{k / m} \tilde{K} \cap$ $F \neq \emptyset$. By the definition of $(m, \alpha)$-neighbourliness it implies that $\alpha \leq a \sqrt{k / m}$, which completes the proof of the lemma.

Returning to the proof of (ii) part of the proposition, recall that the notion of $(m, \alpha)$-neighbourliness for symmetric convex polytopes is invariant under linear isomorphisms of $\mathbb{R}^{k}$. Thus without loss of the generality we may assume that $B_{2}^{k}$ is the ellipsoid of minimal volume containing $K$. By John's theorem (the real case) there exists a subset $J \subset\{1,2, \ldots, n\}$ with $|J| \leq k(k+1) / 2$ such that $B_{2}^{k}$ is the ellipsoid of minimal volume containing $K_{J}:=\operatorname{conv}\left\{ \pm x_{j} \mid j \in J\right\}$. (Indeed, the vertices from $J$ are contact points of $K$ and $B_{2}^{k}$ used in John's decomposition of identity.) This in particular yields $(1 / \sqrt{k}) B_{2}^{k} \subset K_{J}$. Since $n \geq k(k+1) / 2+m$, there exists $I \subset\{1,2, \ldots, n\}$ with $|I|=m$ disjoint from $J$. Thus (ii) follows directly from the lemma.

\section{Technical Proposition}

The proof of Theorem 3.2 is based on the following (uniform) lower estimate for "typical" subgaussian matrices on sets of the form $A B_{1}^{n} \cap S^{n-1}$.

Proposition 6.1 There exist numerical constants $c_{0}^{\prime}, c_{1}^{\prime}>0$ such that the following holds. Let $\gamma$ be a symmetric subgaussian random variable with $\operatorname{Var} \gamma \geq 1$ and the subgaussian constant $\beta$. Let $k \leq n$ be positive integers and $\Gamma$ be a $k \times n$ matrix with independent entries distributed according to $\gamma$. Let $P$ be an orthogonal projection in $\mathbb{R}^{k}$ with rank $P \geq k / 4$. With probability greater than $1-\exp \left(-c_{0}^{\prime} k / \beta^{4}\right)$, we have

$$
|P \Gamma x| \geq \sqrt{k} / 8
$$

for every $x \in A B_{1}^{n} \cap S^{n-1}$, where $A$ is any nonnegative number satisfying

$$
A \leq \frac{c_{1}^{\prime} \sqrt{k}}{\beta^{3} \sqrt{\log \left(n \beta^{4} / c_{1}^{\prime} k\right)}} .
$$

The proof of Proposition 6.1 follows a familiar scheme of " $\varepsilon$-net arguments," with some additional twists related to the geometry of sets $A B_{1}^{n} \cap S^{n-1}$ (cf. [11]).

Let us begin with a standard general remark. For all $x=\left(x_{i}\right) \in S^{n-1}$ and $1 \leq$ $j \leq k$, let $\xi_{j}=\left\langle\Gamma x, e_{j}\right\rangle=\sum_{i} \gamma_{j i} x_{i}$. Then $\xi_{j}$ 's are clearly i.i.d. and symmetric, and a straightforward calculation (that uses the symmetry of the $\gamma_{j i}$ 's) shows that they are subgaussian with $\operatorname{Var} \xi_{j} \geq 1$ and the subgaussian constant at most $\beta$. (This means that $\Gamma x$ is a subgaussian vector in $\mathbb{R}^{k}$.)

We recall two known pointwise estimates. Firstly, the well-known fact for subgaussian vectors (see, e.g., Fact 2.1 in [6]) implies that, for every $x \in S^{n-1}$, we have

$$
\mathbb{P}(|P \Gamma x| \leq u \sqrt{k}) \geq \mathbb{P}(|\Gamma x| \leq u \sqrt{k}) \geq 1-\exp \left(k\left(\log 2-u^{2} / 3 \beta\right)\right)
$$


for every $u>0$.

Secondly, setting rank $P=\ell \geq k / 4$, noting that the Hilbert-Schmidt norm satisfies $\|P\|_{H S}=\sqrt{\ell}$, and using [6], Theorem 2.5, we get, for every $x \in S^{n-1}$,

$$
\mathbb{P}\left(|P \Gamma x| \geq \frac{\sqrt{k}}{4}\right) \geq \mathbb{P}\left(|P \Gamma x| \geq \frac{\|P\|_{H S}}{2}\right) \geq 1-2 \exp \left(-c_{0} k / \beta^{4}\right),
$$

where $0<c_{0} \leq 1$ is a suitable numerical constant.

Proof of Proposition 6.1 The first part of the argument is valid for all $A$. Note that the case $A<1$ is trivial and so fix an arbitrary $A \geq 1$. We shall establish estimates analogous to (6.2) and (6.3) valid for all $x$ in the set of the form $A B_{1}^{n} \cap S^{n-1}$. In fact, in what follows we need only a lower estimate on this set.

For the moment, fix $\varepsilon>0$. We shall find an $\varepsilon$-net (in the Euclidean norm) $\Lambda \subset$ $A B_{1}^{n} \cap S^{n-1}$ with controlled cardinality $|\Lambda|$. Also, we shall find a set $\Lambda^{\prime} \subset 4 B_{2}^{n}$ such that

$$
(2 A / \varepsilon) B_{1}^{n} \cap B_{2}^{n} \subset \operatorname{conv} \Lambda^{\prime},
$$

and again, we will control the cardinality $\left|\Lambda^{\prime}\right|$.

Assume that such $\Lambda$ and $\Lambda^{\prime}$ are already constructed. Consider an arbitrary $x \in$ $A B_{1}^{n} \cap S^{n-1}$. Pick $y_{0} \in \Lambda$ with $\left|x-y_{0}\right| \leq \varepsilon$ and note that

$$
x-y_{0} \in 2 A B_{1}^{n} \cap \varepsilon B_{2}^{n}=\varepsilon\left(\frac{2 A}{\varepsilon} B_{1}^{n} \cap B_{2}^{n}\right) .
$$

Therefore

$$
\left|P \Gamma\left(x-y_{0}\right)\right| \leq\left|\Gamma\left(x-y_{0}\right)\right| \leq \varepsilon \max _{z \in \Lambda^{\prime}}|\Gamma z|,
$$

and thus

$$
|P \Gamma x| \geq \min _{y \in \Lambda}|P \Gamma y|-\varepsilon \max _{z \in \Lambda^{\prime}}|\Gamma z| .
$$

Estimates for the minimum and maximum will be treated separately.

By (6.3) we have

$$
\mathbb{P}\left(\min _{y \in \Lambda}|P \Gamma y| \leq \frac{\sqrt{k}}{4}\right) \leq|\Lambda| 2 \exp \left(-c_{0} k / \beta^{4}\right) .
$$

Take $u=3 \beta$ and recall that $\Lambda^{\prime} \subset 4 B_{2}^{n}$. Hence by (6.2)

$$
\mathbb{P}\left(\max _{z \in \Lambda^{\prime}}|\Gamma z| \geq 12 \beta \sqrt{k}\right) \leq\left|\Lambda^{\prime}\right| \exp (-2 k) .
$$

(Here we used that $3 \beta \geq 3>2+\log 2$.)

In view of (6.5), by combining (6.6) and (6.7) we infer that with probability larger than or equal to

$$
1-|\Lambda| 2 \exp \left(-c_{0} k / \beta^{4}\right)-\left|\Lambda^{\prime}\right| \exp (-2 k)
$$


we have, for all $x \in A B_{1}^{n} \cap S^{n-1}$,

$$
|P \Gamma x| \geq \sqrt{k}(1 / 4-12 \beta \varepsilon) .
$$

Letting $\varepsilon=(96 \beta)^{-1}$, we obtain, with the same probability,

$$
|P \Gamma x| \geq \sqrt{k} / 8
$$

for all $x \in A B_{1}^{n} \cap S^{n-1}$.

In the next step we find sets $\Lambda$ and $\Lambda^{\prime}$ of suitably small cardinalities. To do this, fix for the moment $0<\varepsilon^{\prime} \leq 1$. By Lemma 3.8 in [11], $U_{A^{2} / \delta}$ is an $\varepsilon^{\prime}$-net for $A B_{1}^{n} \cap S^{n-1}$, where $\delta=\left(\varepsilon^{\prime} / 2\right)^{2}$. Set $r=4 A^{2} / \varepsilon^{\prime 2}$. It is known that $U_{r}$ contains an $\varepsilon^{\prime}$-net $\tilde{\Lambda}$ with

$$
|\tilde{\Lambda}| \leq\left(\begin{array}{l}
n \\
r
\end{array}\right)\left(\frac{3}{\varepsilon^{\prime}}\right)^{r} \leq \exp \left(r\left(\log (n / r)+\log \left(3 / \varepsilon^{\prime}\right)\right)\right) .
$$

Clearly, $\tilde{\Lambda}$ is an $2 \varepsilon^{\prime}$-net for $A B_{1}^{n} \cap S^{n-1}$ (but it does not need to be contained in this set). However, a well-known argument shows that there exists $\Lambda \subset A B_{1}^{n} \cap S^{n-1}$ which is a $4 \varepsilon^{\prime}$-net in $A B_{1}^{n} \cap S^{n-1}$ with $|\Lambda| \leq|\tilde{\Lambda}|$. We set $\varepsilon^{\prime}=\varepsilon / 4$, where $\varepsilon$ has been determined just before (6.9).

Note that the set of extreme points of $(2 A / \varepsilon) B_{1}^{n} \cap B_{2}^{n}$ is contained in $(2 A / \varepsilon) B_{1}^{n} \cap$ $S^{n-1}$. Hence by Lemma 4.3 we have

$$
(2 A / \varepsilon) B_{1}^{n} \cap B_{2}^{n} \subset 2 \operatorname{conv} U_{4 A^{2} / \varepsilon^{2}}
$$

This shows that any set $\Lambda^{\prime}$ whose convex hull contains 2 conv $U_{4 A^{2} / \varepsilon^{2}}$ automatically satisfies (6.4). On the other hand, an elementary argument of Lemma 2.3 in [11] shows that there is $\tilde{\Lambda}^{\prime} \subset B_{2}^{n}$ with the property that

$$
\operatorname{conv} 2 \tilde{\Lambda}^{\prime} \supset \operatorname{conv} U_{4 A^{2} / \varepsilon^{2}}
$$

and such that

$$
\left|\tilde{\Lambda}^{\prime}\right| \leq \exp \left(\left(c_{1} A^{2} / \varepsilon^{2}\right) \log \left(c_{1} n \varepsilon^{2} / A^{2}\right)\right)
$$

where $c_{1}>0$ is a numerical constant. Therefore setting $\Lambda^{\prime}=4 \tilde{\Lambda}^{\prime}$, we get a required set with cardinality $\left|\Lambda^{\prime}\right|=\left|\tilde{\Lambda}^{\prime}\right|$ satisfying (6.11).

Our final step is to show that by a suitable choice of universal constants we can ensure that once $A$ admits the upper bound of the Proposition, then the quantity in (6.8) is exponentially close to 1 . First note that we can find universal constants $c_{1}^{\prime}$ and $C_{1}$ such that whenever $A$ satisfies (6.1) (with constant $c_{1}^{\prime}$ ), then each of the cardinalities in (6.10) and (6.11) can be estimated from above by

$$
\exp \left(\left(C_{1} A^{2} / \varepsilon^{2}\right) \log \left(C_{1} n \varepsilon^{2} / A^{2}\right)\right) .
$$

Then recalling that $\varepsilon=1 / 96 \beta$ and decreasing $c_{1}^{\prime}$ if necessary, we can ensure that if $A$ satisfies (6.1), then

$$
\left(C_{1} A^{2} / \varepsilon^{2}\right) \log \left(C_{1} n \varepsilon^{2} / A^{2}\right) \leq c_{0} k /\left(2 \beta^{4}\right),
$$


where $0<c_{0} \leq 1$ is the constant from (6.3). Finally, decreasing $c_{1}^{\prime}$ again, we can ensure that condition (6.1) and the fact that $A \geq 1$ imply that $k \geq \tilde{C} \beta^{6} \geq \tilde{C} \beta^{4}$, where $\tilde{C}$ is a suitable (large) constant chosen in such a way that the bound on $k$ yields that

$$
3 \exp \left(-c_{0} k /\left(2 \beta^{4}\right)\right) \leq \exp \left(-c_{0} k /\left(4 \beta^{4}\right)\right)
$$

Putting these estimates together, we conclude that if $A \geq 1$ satisfies (6.1), then (6.9) holds with probability larger than or equal to

$$
1-\exp \left(-c_{0} k /\left(4 \beta^{4}\right)\right)
$$

This concludes the proof.

\section{Proofs of Theorems}

Proof of Theorem 3.2 Fix an arbitrary $T \in L\left(\mathbb{R}^{k}, \mathbb{R}^{k}\right)$ with $\operatorname{rank} T \geq k / 4$, and let $E=\operatorname{ker} T$. Let $P$ be the orthogonal projection in $\mathbb{R}^{k}$ on the orthogonal complement $F$ of $E$. Then $T$ restricted to $F$ is an isomorphism onto $T(F)$. Let $K \subset \mathbb{R}^{k}$ be a convex symmetric polytope. Note that $T(K)=T P(K)$. Thus $T(K)$ is $(m, \alpha)$-neighbourly if and only if $P(K)$ is $(m, \alpha)$-neighbourly. On the other hand, by Corollary 4.4, one can see that the latter property holds whenever ker $P \Gamma$ is disjoint from $2 \alpha \sqrt{m} B_{1}^{n} \cap$ $S^{n-1}$. Moreover, if this is the case, then $|\mathcal{V}(P K)|=2 n$. By Proposition 6.1 it clearly happens (with probability close to 1 ) for any pair $(m, \alpha)$ satisfying

$$
2 \alpha \sqrt{m} \leq \frac{c_{1}^{\prime} \sqrt{k}}{\beta^{3} \sqrt{\log \left(n \beta^{4} / c_{1}^{\prime} k\right)}} .
$$

This shows the conclusion of the theorem for one operator $T$, and the statement for the family of $N$ operators immediately follows.

Proof of Theorem 3.3 Fix $\sigma \subset\{1,2, \ldots, n\}$ with $|\sigma|=\ell$ and fix for the moment $\Gamma\left(e_{i}\right)$ for $i \in \sigma$. Let $\Gamma_{\sigma}$ be the $k \times(n-\ell)$ matrix resulting from $\Gamma$ after removing columns with indices belonging to $\sigma$. Set $K_{\sigma}=\operatorname{conv}\left\{ \pm \Gamma e_{i} \mid i \notin \sigma\right\}$. Then $P_{\sigma} K=$ $P_{\sigma} K_{\sigma}$. Since $k<n-\ell$, we may apply Theorem 3.2 to the random matrix $\Gamma_{\sigma}$ and a single operator $P_{\sigma}$. It follows that the conditional probability (with $\left\{\Gamma e_{i} \mid i \in \sigma\right\}$ fixed) that the polytope $P_{\sigma} K_{\sigma}$ is $(m, \alpha)$-neighbourly for $m$ and $\alpha$ satisfying (3.1) and the cardinality of the set of vertices is equal to $2(n-\ell)$ is greater than $1-$ $\exp \left(-c_{1} k / \beta^{4}\right)$. By integrating with respect to vectors $\Gamma\left(e_{i}\right)$ for $i \in \sigma$ we infer that the polytope $P_{\sigma} K$ is $(m, \alpha)$-neighbourly with the same probability, and the theorem easily follows.

Proof of Theorem 3.4 We begin with the following easy lemma. 
Lemma 7.1 There is a constant $\rho>0$ such that, for all $k \geq 4$ and $x, y \in \mathbb{R}^{k}$, letting $a=\max \{|x|,|y|\}$, one has

$$
\begin{aligned}
v_{k}\{U \in O(k)|| U x+y \mid \geq a / 2\} & =v_{k}\{U \in O(k)|| x+U y \mid \geq a / 2\} \\
& \geq 1-\exp (-\rho k) .
\end{aligned}
$$

Proof The equality is obvious. To prove the inequality, note that without loss of generality we may assume that $a=|x|=1$. Clearly

$$
v_{k}\{U \in O(k)|| U x+y \mid<1 / 2\}=\tilde{v}_{k-1}\left\{z \in S^{k-1}|| z+y \mid<1 / 2\right\},
$$

where $\tilde{v}_{k-1}$ denotes the normalized Haar measure on $S^{k-1}$. The latter set is a cap on the sphere (which might be empty, as is in the case $|y| \leq 1 / 2$ ) whose measure is maximal if $|y|=\sqrt{3} / 2$. Thus the lemma is concluded by recalling the well-known estimate for the measure of the cap

$$
\left\{z \in S^{k-1} \mid\left\langle z, e_{1}\right\rangle>\sqrt{3} / 2\right\} .
$$

Returning to the proof of the theorem, consider the $k \times 2 n$ matrix $\Gamma_{U}$ with $j$ th column equal to $\Gamma\left(e_{j}\right)$ for $j \leq n$ and equal to $U \Gamma\left(e_{i}\right)$ for $j=n+i, i=1,2, \ldots, n$. Then $K_{U}=\operatorname{conv}\left\{ \pm \Gamma_{U}\left(e_{i}\right) \mid i=1,2, \ldots, 2 n\right\}$.

For $z=\left(a_{1}, a_{2}, \ldots, a_{2 n}\right) \in S^{2 n-1}$, set $z_{1}=\left(a_{1}, a_{2}, \ldots, a_{n}\right)$ and $z_{2}=\left(a_{n+1}, a_{n+2}\right.$, $\left.\ldots, a_{2 n}\right)$. Since

$$
\left|\Gamma_{U}(z)\right|=\left|\Gamma\left(z_{1}\right)+U \Gamma\left(z_{2}\right)\right| \leq\left|\Gamma\left(z_{1}\right)\right|+\left|U \Gamma\left(z_{2}\right)\right|=\left|\Gamma\left(z_{1}\right)\right|+\left|\Gamma\left(z_{2}\right)\right|,
$$

for every $C>0$, the inequality $\left|\Gamma_{U}(z)\right|>2 C$ implies

$$
C<\max \left\{\left|\Gamma\left(z_{1}\right)\right|,\left|\Gamma\left(z_{2}\right)\right|\right\} \leq \max \left\{\left|\Gamma\left(z_{1}\right)\right| /\left|z_{1}\right|,\left|\Gamma\left(z_{2}\right)\right| /\left|z_{2}\right|\right\} .
$$

Hence by (6.2), for every $z \in S^{2 n-1}$ and every $u>0$, we have

$$
\tilde{\mathbb{P}}\left(\left|\Gamma_{U}(z)\right| \leq 2 u \sqrt{k}\right) \geq 1-2 \exp \left(k\left(\log 2-u^{2} / 3 \beta\right)\right) .
$$

(Recall that the probability $\tilde{\mathbb{P}}$ was defined before the statement of Theorem 3.4.)

On the other hand, note that $\max \left\{\left|z_{1}\right|,\left|z_{2}\right|\right\} \geq 1 / \sqrt{2}$. Thus, using the right-hand side inequality in (6.3) for $P=I d$, it follows that, for every $z \in S^{2 n-1}$,

$$
\mathbb{P}\left(\max \left\{\left|\Gamma\left(z_{1}\right)\right|,\left|\Gamma\left(z_{2}\right)\right|\right\} \geq \frac{\sqrt{k}}{2 \sqrt{2}}\right) \geq 1-2 \exp \left(-c_{0} k / \beta^{4}\right) .
$$

Combining this with Lemma 7.1, it follows that, for every $z \in S^{2 n-1}$,

$$
\begin{aligned}
\tilde{\mathbb{P}}\left(\left|\Gamma_{U}(z)\right| \geq \sqrt{k} / 4 \sqrt{2}\right) & =\tilde{\mathbb{P}}\left(\left|\Gamma\left(z_{1}\right)+U \Gamma\left(z_{2}\right)\right| \geq \sqrt{k} / 4 \sqrt{2}\right) \\
& \geq 1-2 \exp \left(-c_{0} k / \beta^{4}\right)-\exp (-\rho k) .
\end{aligned}
$$

We also need the following analog of Proposition 6.1. 
Proposition 7.2 There exist numerical constants $\tilde{c}, c_{1}^{\prime}>0$ such that the following holds. Let $\gamma$ be a symmetric subgaussian random variable with $\operatorname{Var} \gamma \geq 1$ and the subgaussian constant $\beta$. Let $k \leq n$ be positive integers and $\Gamma$ be a $k \times n$ matrix with independent entries distributed according to $\gamma$. Let $\Gamma_{U}$ be as defined above. With probability $\tilde{\mathbb{P}}$ greater than $1-\exp \left(-\tilde{c} k / \beta^{4}\right)$, we have

$$
\left|\Gamma_{U} x\right| \geq \sqrt{k} / 8 \sqrt{2}
$$

for every $x \in A B_{1}^{n} \cap S^{n-1}$, where $A$ is any nonnegative number satisfying

$$
A \leq \frac{c_{1}^{\prime} \sqrt{k}}{\beta^{3} \sqrt{\log \left(n \beta^{4} / c_{1}^{\prime} k\right)}} .
$$

The proof of the proposition goes exactly the same line of argument as the proof of Proposition 6.1 (with suitable adjustment of constants and with (7.1) and (7.2) replacing (6.2) and (6.3)). We left the details to the reader.

Clearly Theorem 3.4 is an obvious consequence of Proposition 7.2 and Corollary 4.4.

Acknowledgement The authors wish to thank the anonymous referee for suggestions that helped to improve the presentation of the paper.

\section{References}

1. Bárány, I., Pór, A.: On $0-1$ polytopes with many facets. Adv. Math. 161, 209-228 (2001)

2. Gatzouras, D., Giannopoulos, A.: Threshold for the volume spanned by random points with independent coordinates. Isr. J. Math. (2008, to appear)

3. Gatzouras, D., Giannopoulos, A., Markoulakis, N.: Lower bound for the maximal number of facets of a 0/1 polytope. Discrete Comput. Geom. 34, 331-349 (2005)

4. Gluskin, E.: The diameter of the Minkowski compactum is roughly equal to $n$. Funkc. Anal. Priloz. 15, 72-73 (1981). (Russian)

5. Grünbaum, B.: Convex Polytopes, 2nd edn. Graduate Texts in Mathematics, vol. 221. Springer, New York (2003)

6. Latała, R., Mankiewicz, P., Oleszkiewicz, K., Tomczak-Jaegermann, N.: Banach-Mazur distances and projections on random subgaussian polytopes. Discrete Comput. Geom. 38, 29-50 (2007)

7. Litvak, A.E., Pajor, A., Rudelson, M., Tomczak-Jaegermann, N.: Smallest singular value of random matrices and geometry of random polytopes. Adv. Math. 195, 491-523 (2005)

8. Mankiewicz, P., Tomczak-Jaegermann, N.: Solution of the finite-dimensional homogeneous Banach space problem. Isr. J. Math. 75, 129-159 (1991)

9. Mankiewicz, P., Tomczak-Jaegermann, N.: Quotients of finite-dimensional Banach spaces; random phenomena. In: Johnson, W.B., Lindenstrauss, J. (eds.) Handbook of the Geometry of Banach Spaces, vol. II, pp. 1201-1246. North-Holland, Amsterdam (2003)

10. Mendelson, S., Pajor, A., Tomczak-Jaegermann, N.: Reconstruction and subgaussian operators in asymptotic geometric analysis. Geom. Funct. Anal. 17, 1248-1282 (2007)

11. Mendelson, S., Pajor, A., Tomczak-Jaegermann, N.: Uniform uncertainty principle for Bernoulli and subgaussian ensembles. Constr. Approx. (2008, to appear)

12. McMullen, P., Shepard, G.C.: Diagrams for centrally symmetric polytopes. Mathematika 15, 123-138 (1968)

13. Schneider, R.: Neighbourliness of centrally symmetric polytopes in high dimensions. Mathematika 22, 176-181 (1975)

14. Szarek, S.J.: The finite-dimensional basis problem with an appendix on nets of Grassman manifolds. Acta Math. 151, 153-179 (1983) 